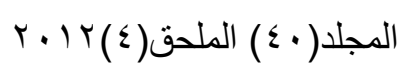

\section{الإقراض الزراعي القصير الأجل و أثره في الوصول الى حجم الانتاج الأمثل لاى مزارعي القمح في محافظة نينوى الرى \\ هناء سلطان داؤد \\ قسم الاقتصاد الزر اعي/ كلية الزر اعة والغابات/ جامعة الموصل}

\section{الخلاصة}

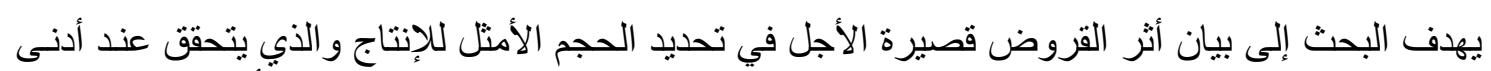

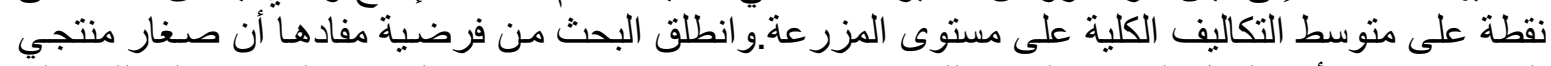

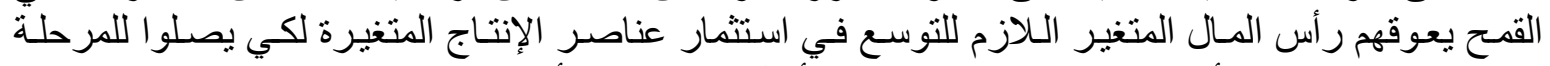

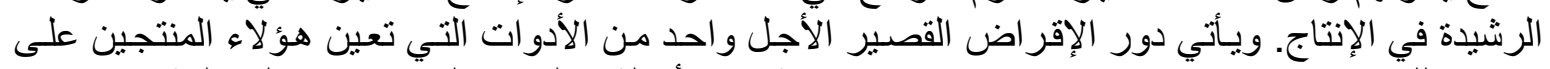

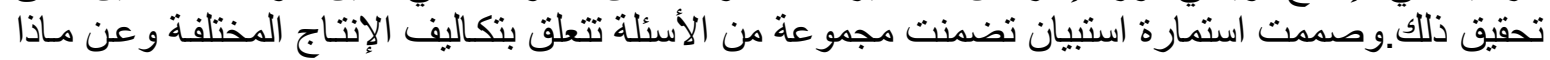

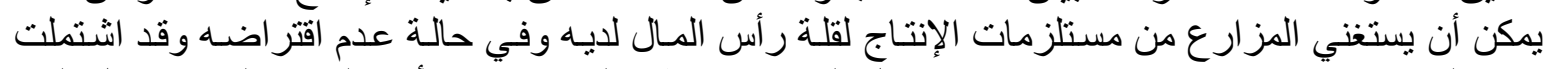

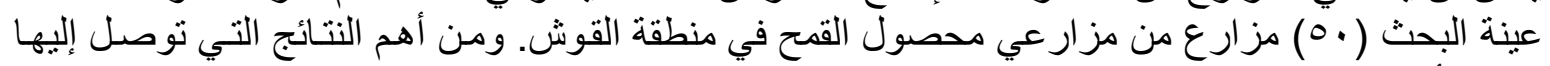

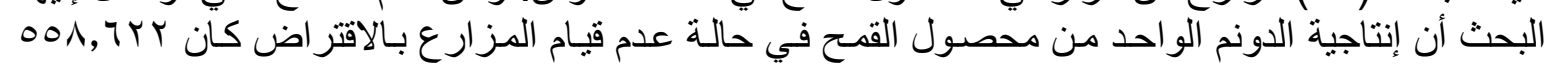

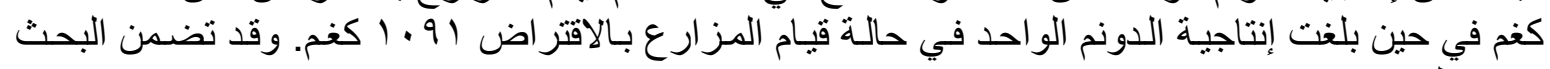
بعض التوصيات.

\section{المقدمة}

يعاني المزارع في العراق شأنه في ذلك شان المزارعين في معظم البلدان النامية من مشكلة عدم كفاية

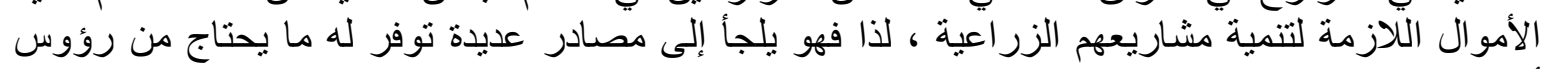

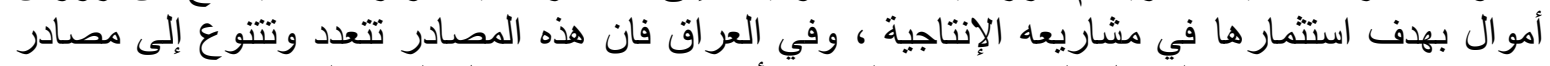

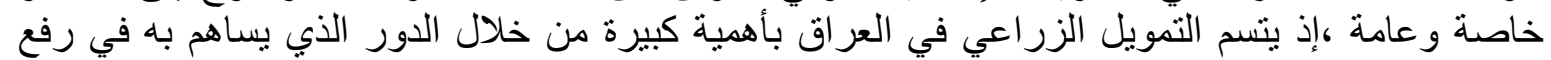

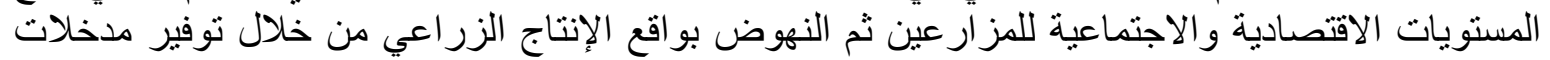

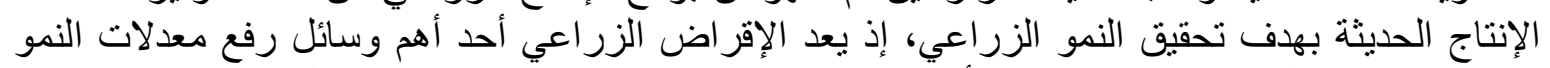

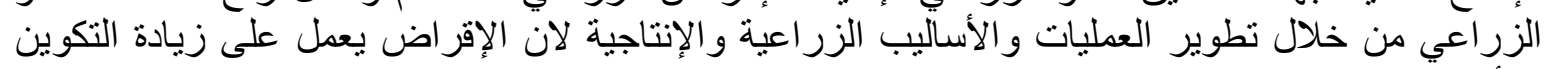

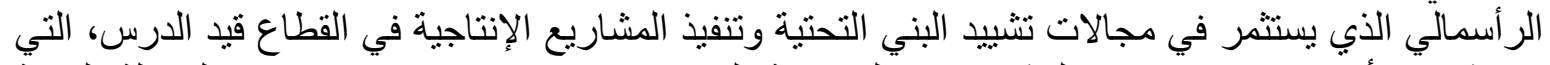

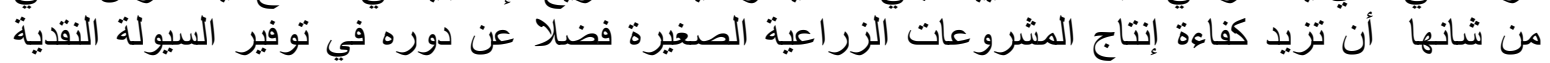

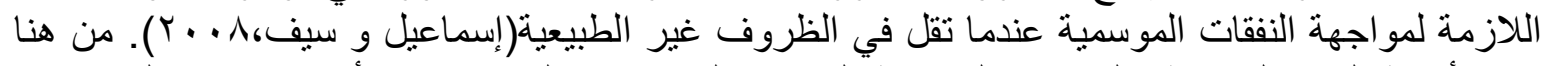

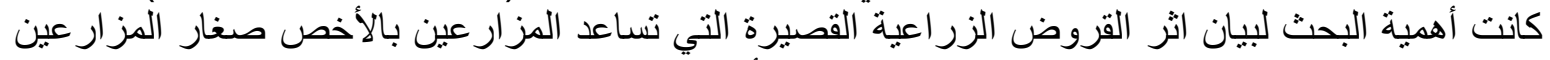

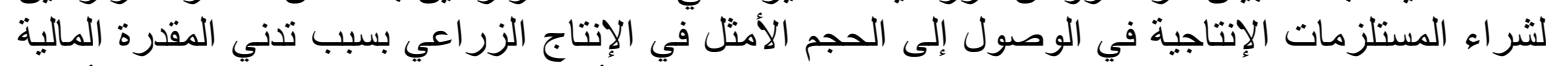

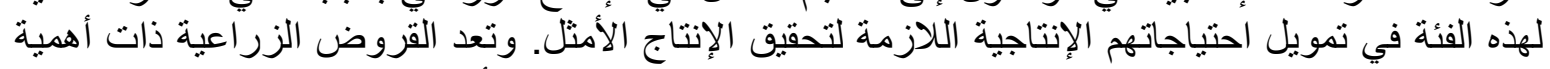

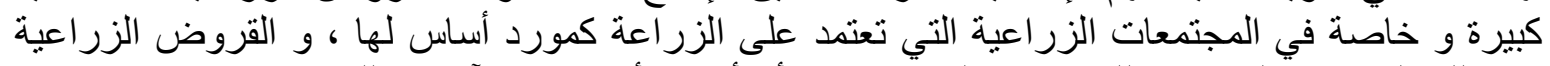

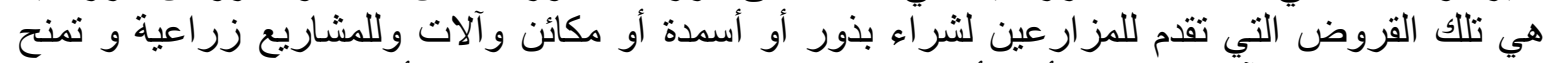

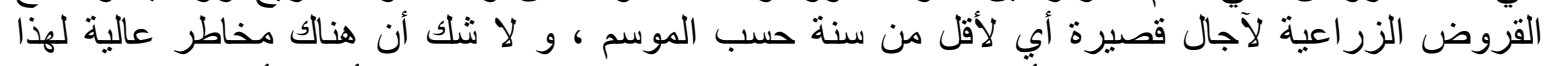

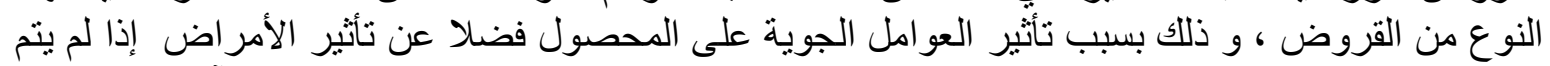

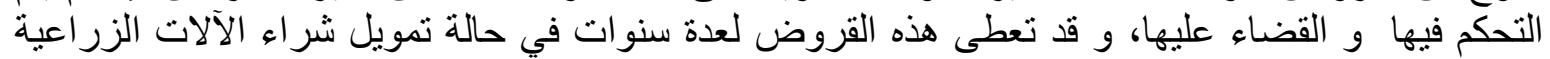

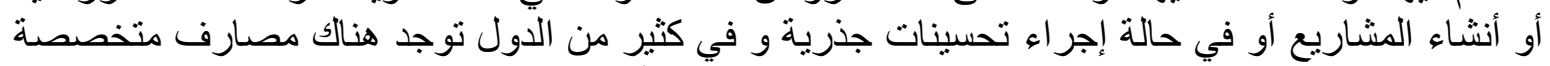

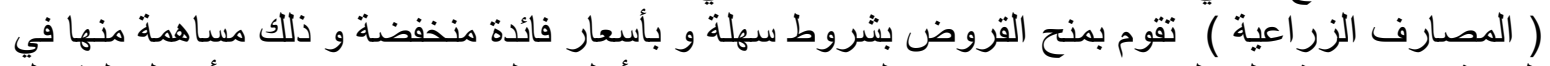

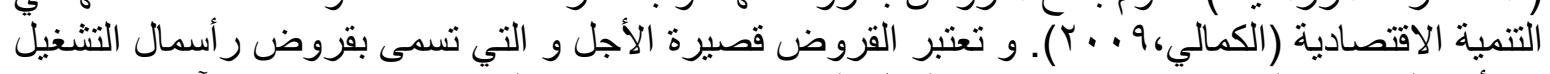

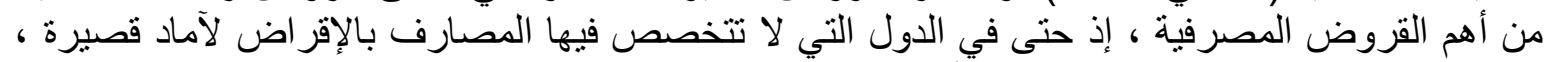

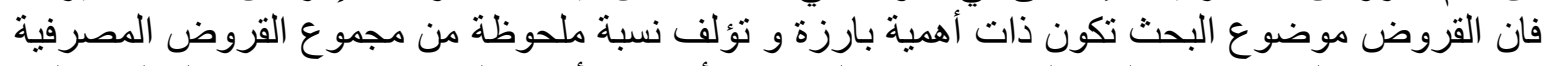

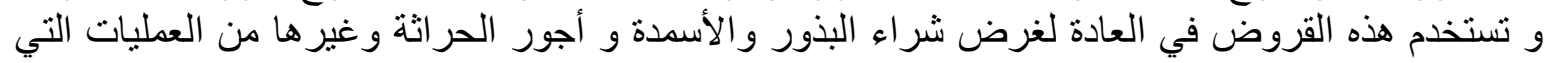

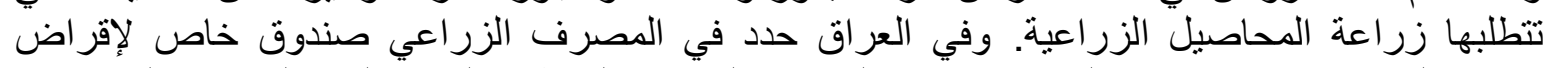

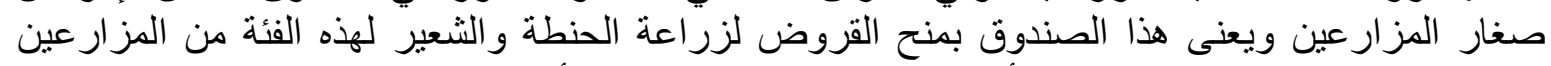

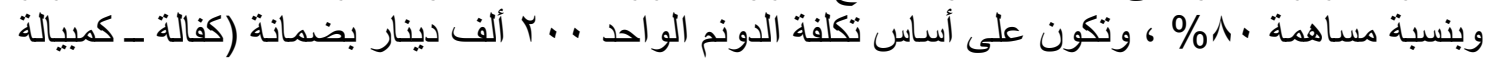
ـ ضمانة عقارية) ويصرف القرض ويسترد بمدة لا تزيد عن سنة (مجهول، ـ ( • ب ). وتتحدد مشكلة البحث 


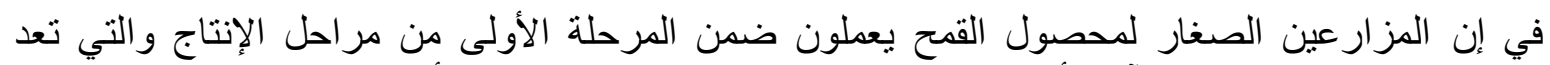

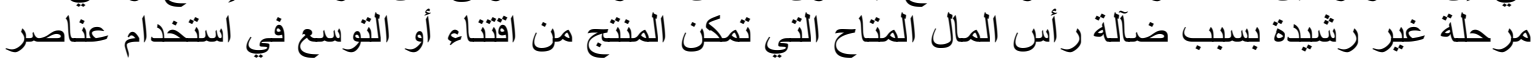

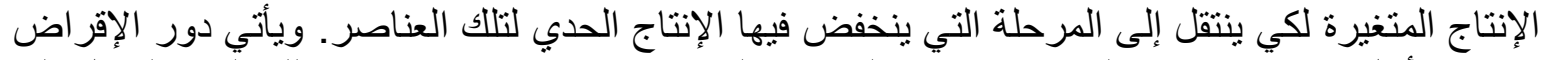

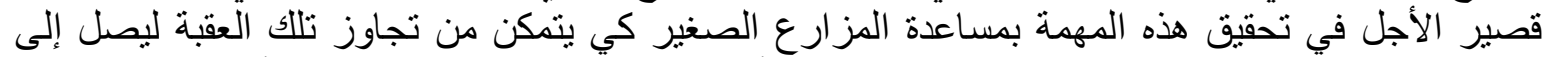

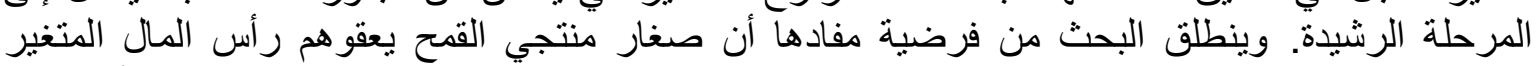

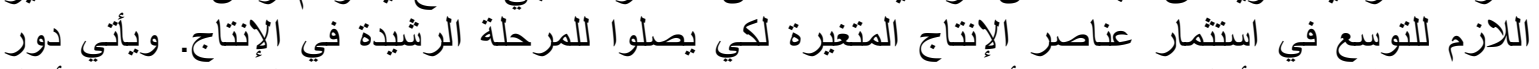

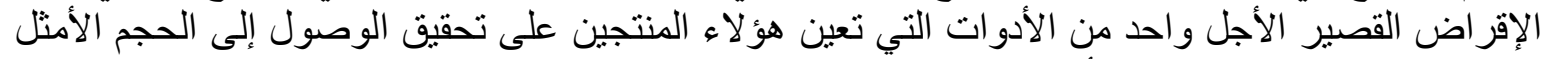

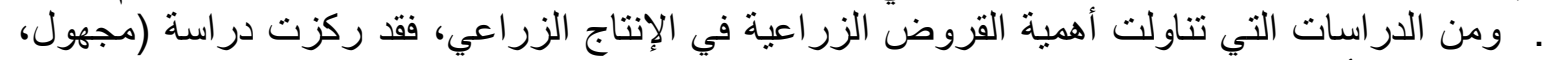

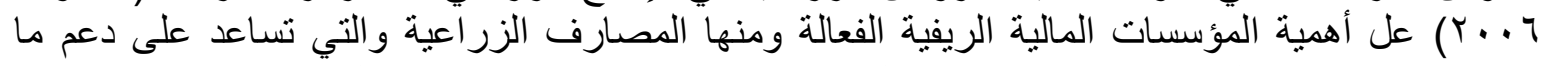

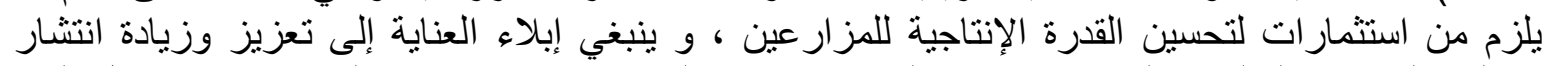

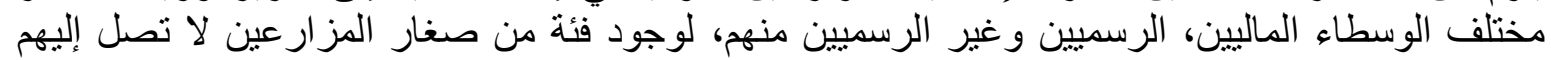

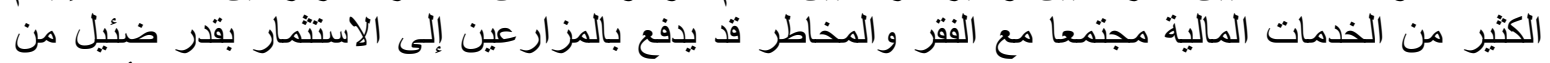

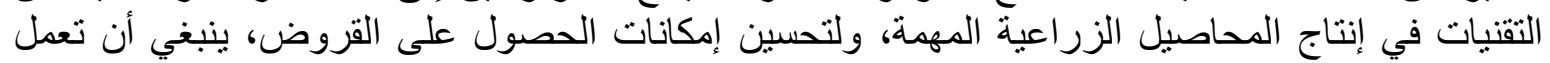

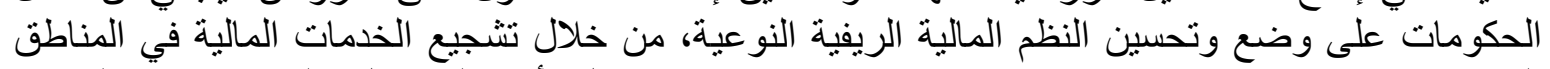

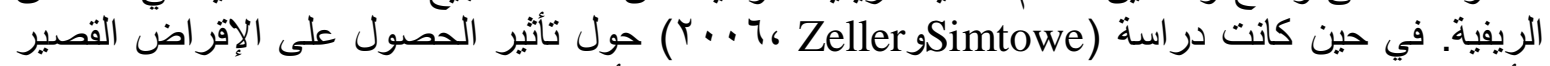

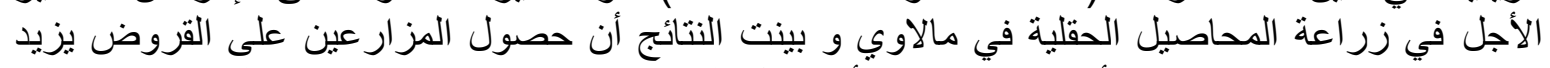

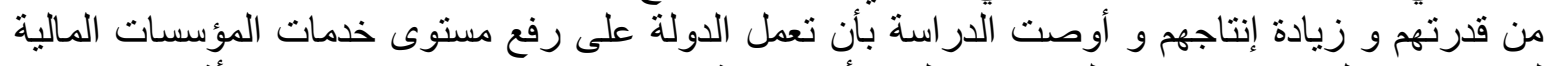

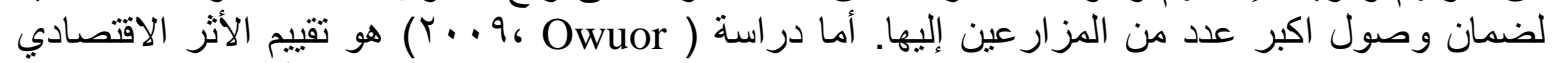

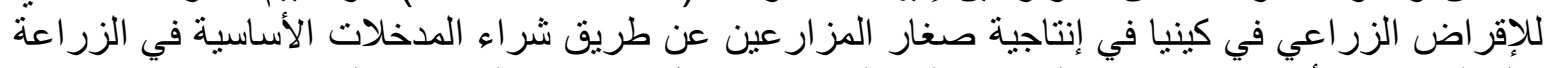

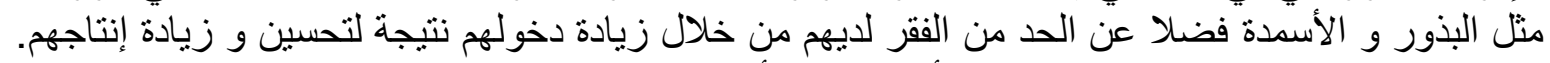

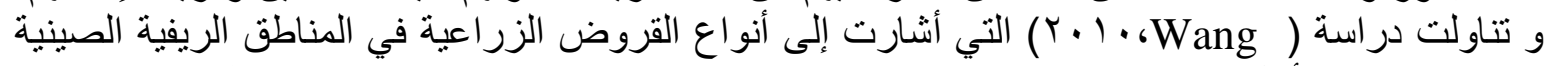

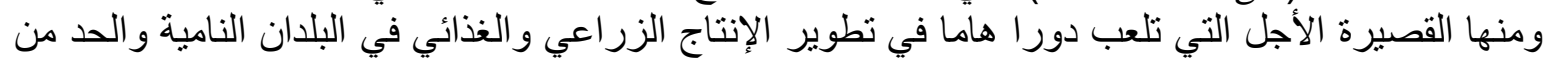

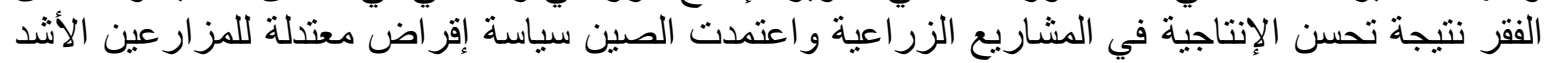

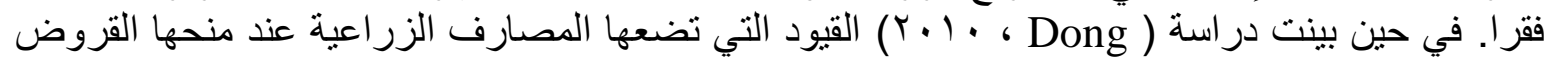

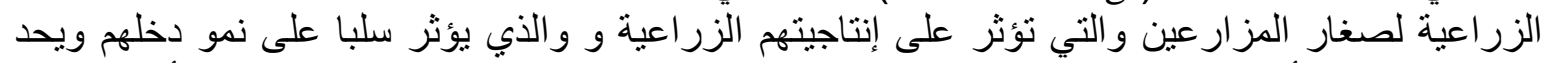

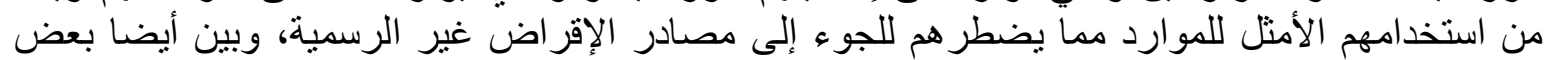

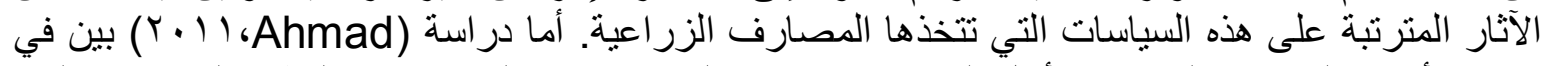

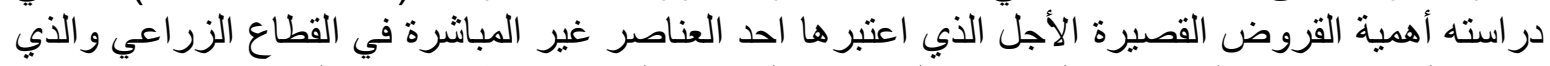

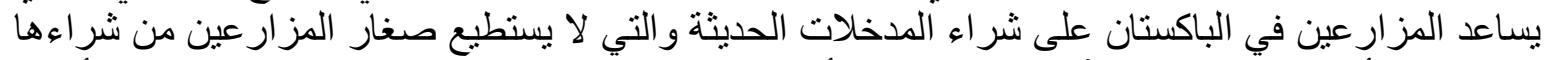

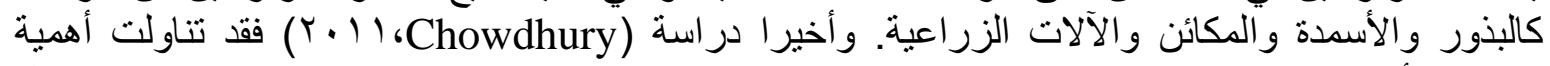

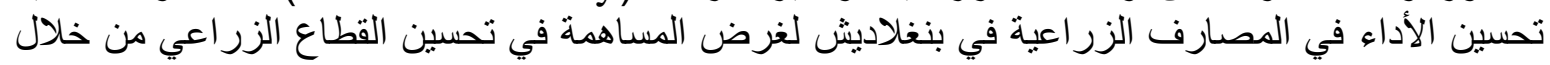

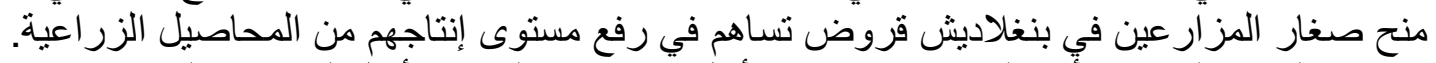

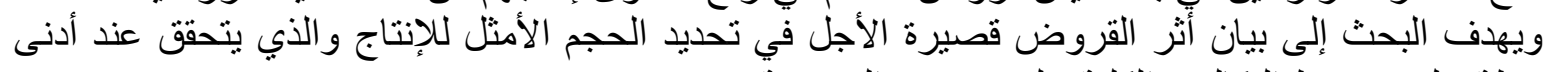

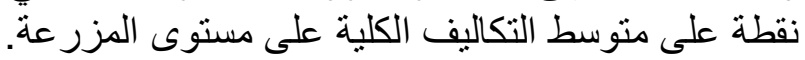

\section{مواد البحث وطرائقه}

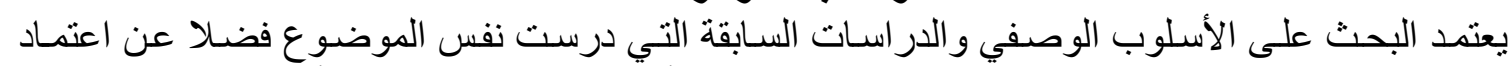

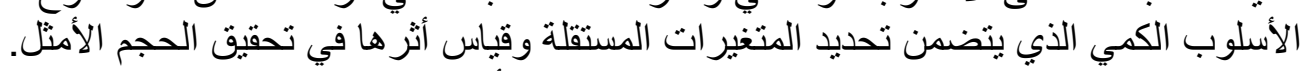

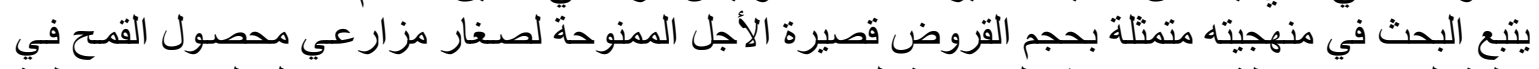

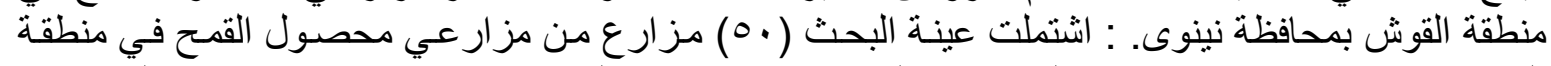

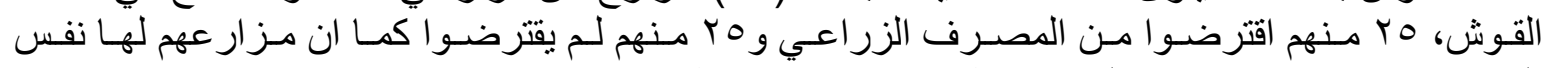

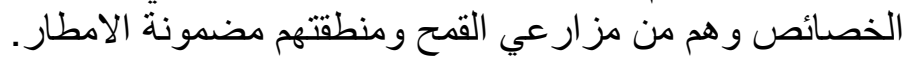

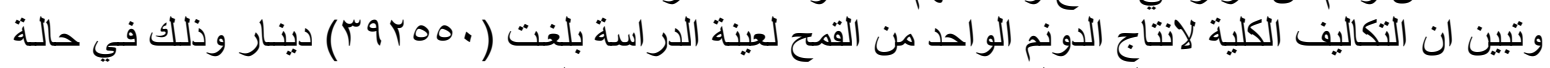

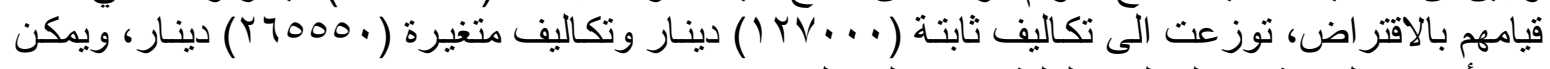

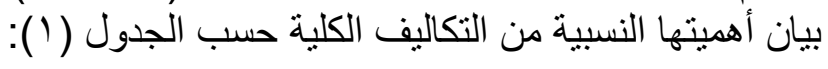




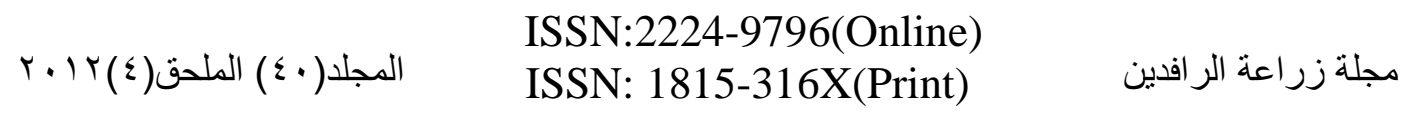

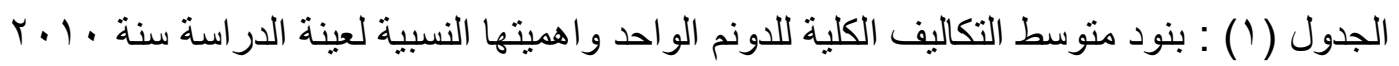

\begin{tabular}{|c|c|c|}
\hline النسبة\% & تكاليف انتاج الدونم الواحد بالدينار & بنود التكاليف \\
\hline rr & IrV... & التكاليف الثابتة \\
\hline 71 & Y7000. & التكاليف المتغيرة \\
\hline $1 \cdots$ & rqYo0. & المجموع \\
\hline
\end{tabular}

المصدر: احتسبت بالاعتماد على بيانات أستمارة الاستبيان

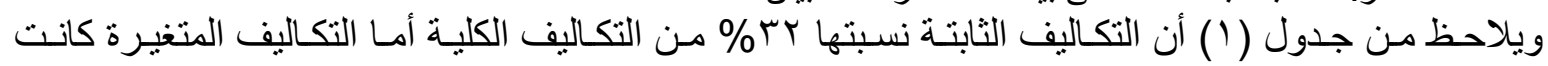

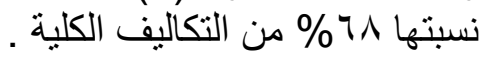

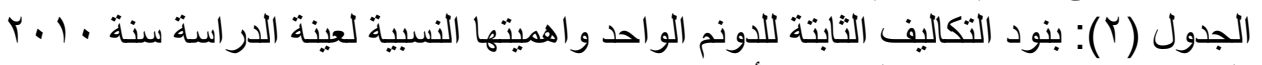
المصدر : احتسبت بالاعنماد على بيانات أستمارة الاستبيان

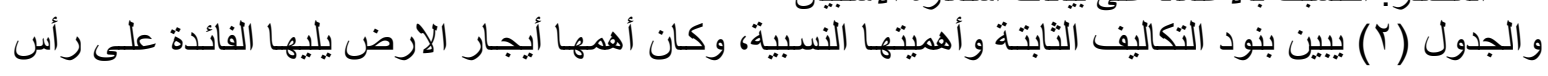

\begin{tabular}{|c|c|c|}
\hline النسبة\% & القيمة بالدينار & بنود التكاليف الثابتة \\
\hline 199 & $1 \ldots \ldots$ & ايجار الارض \\
\hline 9 & $1, \ldots$ & الفائدة على رأس المال \\
\hline IT & $10 \ldots$ & العمل العائلي \\
\hline $1 \cdots$ & ITV... & المجموع \\
\hline
\end{tabular}

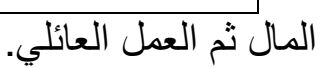

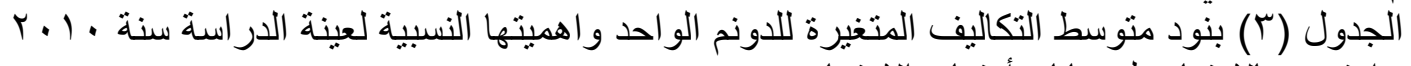

المصدر : احتسبت بالاعتماد على بئن بيات أستمارة الاستبيان وجدول (r) يبين بنود التكاليف المتغيرة وأهميتها النسبية، وكان أهم بند هو قيمة الاستمدة يليـه العمل الآلي ثم

\begin{tabular}{|c|c|c|}
\hline النسبة\% & القيمة بالدينار & بنود التكاليف المتغيرة \\
\hline Ir & $r .00$ & بذور \\
\hline Tr & $10 \ldots$ & اسمدة \\
\hline TA & vo... & العمل الالي \\
\hline 9 & ro... & العمل الاجير \\
\hline 19 & $0 \ldots$. & الحر اثثة \\
\hline $1 \cdots$ & r7000. & المجموع \\
\hline
\end{tabular}

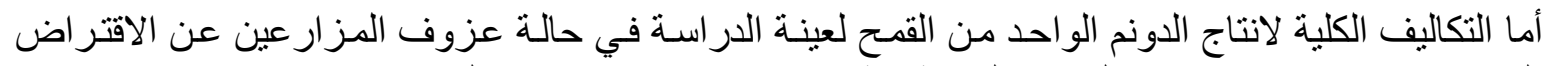

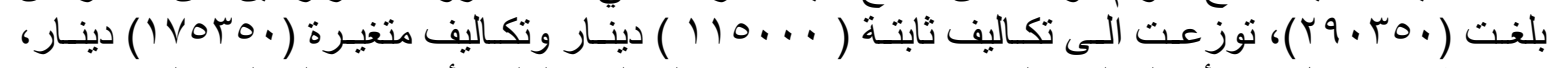

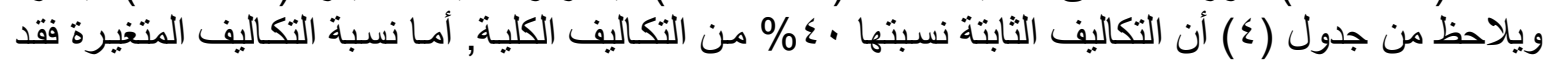

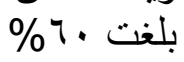

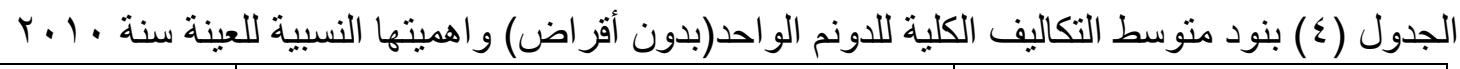

\begin{tabular}{|c|c|c|}
\hline النسبة\%\% & تكاليف انتاج الدونم الواحد بالدينار & بنود التكاليف \\
\hline$\varepsilon$ & $110 \ldots$ & التكاليف الثابتة \\
\hline 7. & IVOro. & التكاليف المتغيرة \\
\hline $1 \ldots$ & rq. ro. & المجموع \\
\hline
\end{tabular}

المصدر: احتسبت بالاعتماد على بيانات أستمارة الاستبيان 


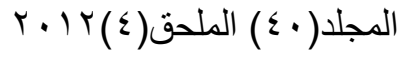
ISSN:2224-9796(Online)
ISSN: 1815-316X(Print)
مجلة زر اعة الر افدين

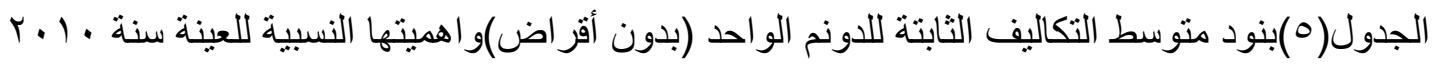

\begin{tabular}{|c|c|c|}
\hline النسبة\%\% & القيمة بالدينار & بنود التكاليف التابتة \\
\hline$\wedge \vee$ & $1 \ldots$ & ايجار الارض \\
\hline$\varepsilon$ & $0 \ldots$ & الفائدة على ر أس المال \\
\hline 9 & $1 \ldots$ & العمل العائلي \\
\hline $1 \cdots$ & $110 \ldots$ & المجموع \\
\hline
\end{tabular}

المصدر: أحتسيت بالاعتماد على بيانات أستمارة الاستبيان

وجدول (0) يبين بنود التكاليف الثابتة وأهميتها النسبية، وكانيان وكان أهمها أيجار الارض بلينها الفائدة على رأس المال ثم العمل العائلي.

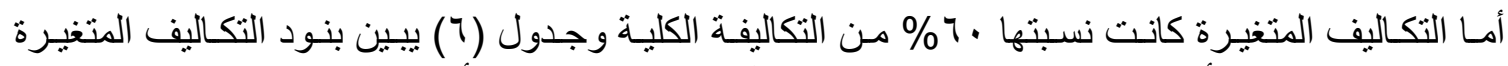

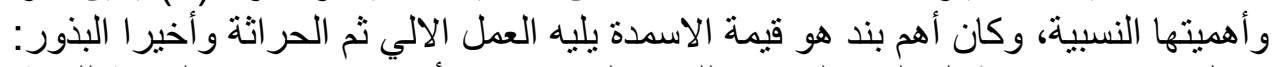

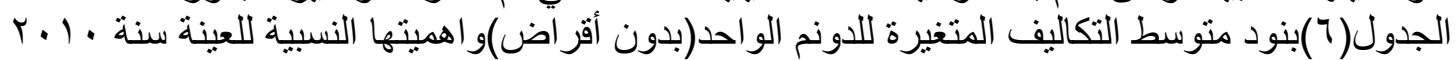

\begin{tabular}{|c|c|c|}
\hline النسبة\% & القيمة بالدينار & بنود التكاليف المتغيرة \\
\hline $1 \leq$ & roro. & بذور \\
\hline rV & $70 \ldots$ & اسمدة \\
\hline rq & $0 \ldots$ & العمل الالي \\
\hline$r$. & ro... & الحراثة \\
\hline $1 \ldots$ & IVoro. & المجموع \\
\hline
\end{tabular}

المصدر: أحتسيت بالاعتماد على بيانات أستمارة الاستبيان

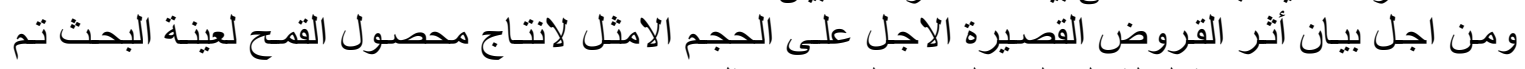

$\mathrm{TC}=\mathrm{a}+\mathrm{b}_{1} \mathrm{X}_{1}$

$T C=a+b_{1} X_{1}+b_{2} X_{1}^{2}$

$\mathrm{TC}=\mathrm{a}+\mathrm{b} 1 \mathrm{X} 1+\mathrm{b}_{2} \mathrm{X}_{1}{ }^{2}+\mathrm{b}_{3} \mathrm{X}_{1}{ }^{3}$

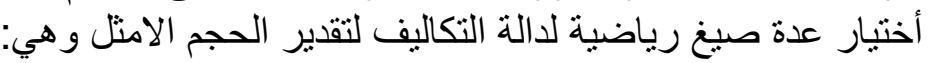
الصيغة الخطية الصيغة التربيعية الصيغة التكعيبية TC

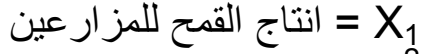
=X $X_{1}{ }^{2}$ معامل = X $_{1}{ }^{3}$

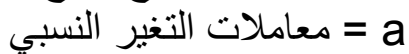

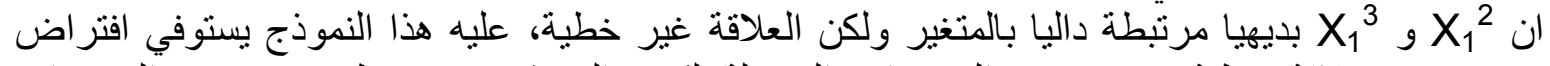

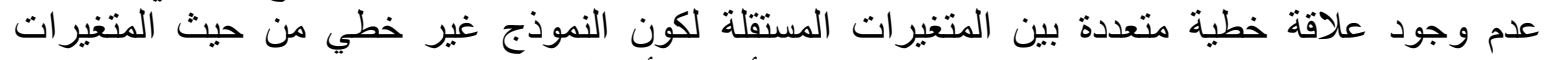

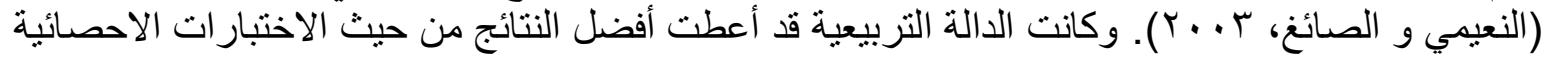
و القياسية والمتفقة مع النظرية الاقتصادية.

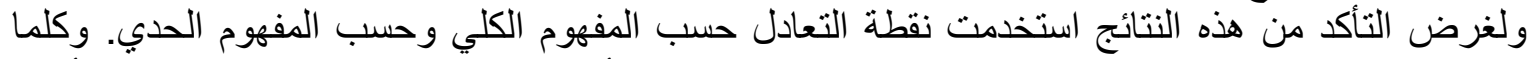
انخفضت نقطة التعادل كلما ارتفعت فرص المزان النفارع في تحقيق الأرباح من خلال الوصول التى حجم الأنتاج

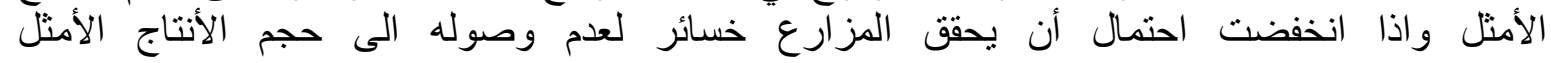

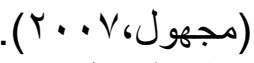
نقطة التعادل حسب المفهوم الكلي: وفق هذا المفهوم تتحدد نقطة التعادل بتساوي التكلفة الكلية مع الايرادات $\mathrm{TC}=\mathrm{TR}$ الكلية 


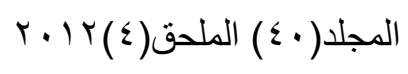

$$
\begin{aligned}
& \text { ISSN:2224-9796(Online) } \\
& \text { ISSN: 1815-316X(Print) } \\
& \text { مجلة زر اعة الر افدين } \\
& \mathrm{TC}=\mathrm{b}_{\mathrm{o}}+\mathrm{b}_{1} \mathrm{Q} \\
& \mathrm{TR}=\mathrm{P} \cdot \mathrm{Q} \\
& \mathrm{P}=\mathrm{b}_{\mathrm{o}}-\mathrm{b}_{1} \mathrm{Q} \\
& \mathrm{TR}=\left(\mathrm{b}_{\mathrm{o}}-\mathrm{b}_{1} \mathrm{Q}\right) \mathrm{Q} \\
& \mathrm{TR}=\mathrm{b}_{\mathrm{o}} \mathrm{Q}-\mathrm{b}_{1} \mathrm{Q}^{2} \\
& \mathrm{TC}=\mathrm{TR} \\
& b_{o}+b_{1} Q=b_{o} Q-b_{1} Q^{2} \\
& \text { TFC = التكاليف الكلية الثابتة } \\
& \text { TVC } \\
& \text { ودالة التكاليف الكلية هي دالة في كمية الانتاج: } \\
& \text { = كمية الانتاج = Q } \\
& \text { أما دالة الاير اد الكلي فهي: } \\
& \text { و سعر وحدة الانتاج } \\
& \text { وبما ان السعر دالة في الكمية فأن: } \\
& \text { و عند نقطة التعادل: }
\end{aligned}
$$

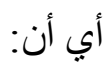

$$
\begin{aligned}
& \text { = }=b_{0} \\
& \text { و = bعامل التكاليف المتغيرة = } \\
& \mathrm{Y}=\mathrm{b}_{\mathrm{o}} / 1-\mathrm{b}_{1} \mathrm{Q} / \mathrm{b}_{\mathrm{o}} \mathrm{Q}-\mathrm{b}_{1} \mathrm{Q}^{2} \\
& \text { ومن المعادلة الاخيرة تحسب نقطة التعادل بالمجاميع النقاية و كالاتي: } \\
& \text { و = نقطة التعادل بالمجاميع النقدية } \\
& \text { وبنفس المفهوم تحسب نقطة التعادل: } \\
& \text { = الاير اد الحدي = MR } \\
& \text { MC } \\
& \mathrm{MR}=\mathrm{dTR} / \mathrm{dQ} \\
& \text { ويمكن الحصول على MR بأخذ المشتقة الجزئية لدالة التكلفة الكلية بالنسبة للأنتاج وكالآتي: } \\
& \mathrm{MC}=\mathrm{dTC} / \mathrm{dQ} \\
& \text { ومن المعادلتين أعلاه يمكن الحصول على نقطة التعادل بالمجاميع الكمية (النعيمي و غز ال، . . Y) } \\
& \text { Y= TFC/ MR-MC } \\
& \text { النتائج و المناقشة المزارة } \\
& \mathrm{TC}=39284+351 \mathrm{Q}+0.033 \mathrm{Q}^{2} \\
& \text { ا . حجم إنتاج الدونم الواحد الذي يصل إليه المزارع عندما يحصل على قرض : } \\
& \mathrm{t}=\quad \begin{array}{lll}
3.025 & 6.420 & 3.302
\end{array} \\
& \mathrm{R}^{2}=80.4 \quad \mathrm{R}^{-2}=82.1 \quad \mathrm{~F}=50.36 \mathrm{D}-\mathrm{W}=2.25 \\
& =\mathrm{MC}=351+0.067 \mathrm{Q} \frac{d T c}{d Q} \\
& \mathrm{AC}=\stackrel{39284+351 \mathrm{Q}+0.033 \mathrm{Q}^{2}}{ }
\end{aligned}
$$

\section{Y= TFC/ 1- TVC/TR}




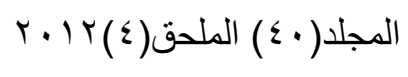
ISSN:2224-9796(Online)
ISSN: 1815-316X(Print)
مجلة زر اعة الر افدين

$$
\begin{aligned}
& \mathrm{MC}=\mathrm{AC} \\
& 1340+0.066 \mathrm{Q}=\frac{\mathrm{Q}}{\mathrm{Q}} \\
& \therefore \mathrm{Q}=1091 \mathrm{Kg} \frac{39284}{0.033} \mathrm{Q}^{2}=
\end{aligned}
$$

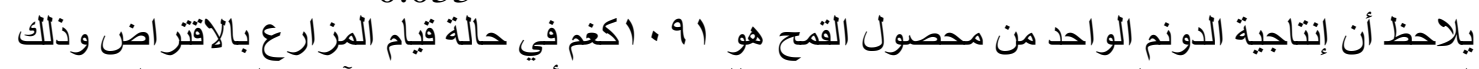
لقيامه باستخدام حبوب القمح ذات مو اصفات جيدة كذللك استخدامه أسمدة جيدة والآلات الحيثة الحات بالحر اثثة.

$$
\begin{aligned}
& \text { r. حجم إنتاج الدونم الواحد الذي يصل إليه المزارع عندما يعزف عن الاقراض: } \\
& \mathrm{TC}=21202+500 \mathrm{Q}+0.0680 \mathrm{Q}^{2} \\
& \mathrm{t}=2.531 \quad 3.102 \quad 2.911 \\
& \mathrm{R}^{2}=79.5 \quad \mathrm{R}^{-2}=82.1 \quad \mathrm{~F}=32.10 \mathrm{D}-\mathrm{W}=2.14 \\
& \frac{d T c}{d Q}=\mathrm{MC}=500+0.136 \mathrm{Q} \\
& \mathrm{AC}=\frac{21202+500 \mathrm{Q}+0.068 \mathrm{Q}^{2}}{\mathrm{Q}} \\
& \mathrm{MC}=\mathrm{AC} \\
& 500+0.136 \mathrm{Q}=\frac{21202+500 \mathrm{Q}+0.068 \mathrm{Q}^{2}}{\mathrm{Q}} \\
& \therefore \mathrm{Q}=558.622 \mathrm{Kg} \quad \frac{21202}{0.068} \mathrm{Q} 2=
\end{aligned}
$$

\section{$\mathbf{Y}=\mathbf{T F C} / 1-\mathrm{TVC} / \mathrm{TR}$}

$\mathrm{Y}=127000 / 1-265550 /(650)(1091)$

$\mathrm{Y}=127000 / 1-0.374$

$\mathrm{Y}=202875.39$

Y. نقطة التعادل حسب المفهوم الحدي:

$$
\begin{aligned}
& \mathbf{Y}=\mathbf{T F C} / \mathbf{M R}-\mathbf{M C} \\
& \mathrm{Y}=127000 / \mathrm{MR}-\mathrm{MC} \\
& \mathrm{MR}=650 \\
& \mathrm{MC}=351+0.067 \mathrm{Q} \\
& \mathrm{MC}=351+(0.067 \times 1091)=424.097 \\
& \mathrm{Y}=127000 / 650-424.097 \\
& \mathrm{Y}=562.189
\end{aligned}
$$




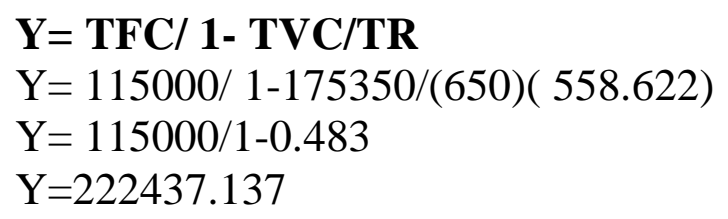

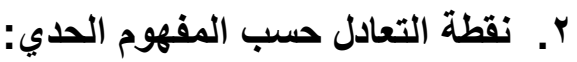

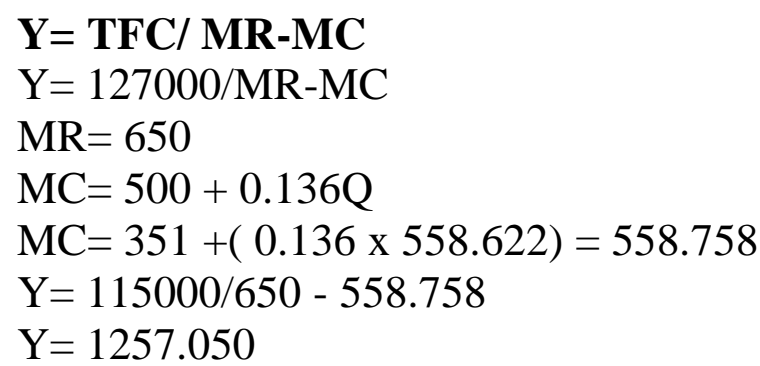

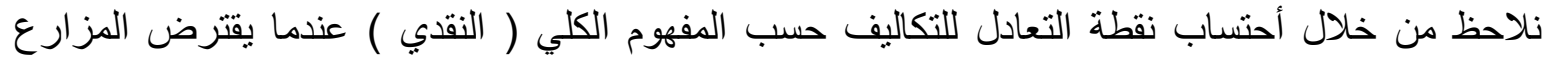

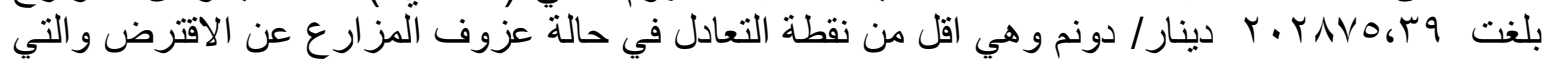

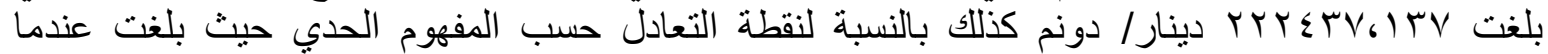

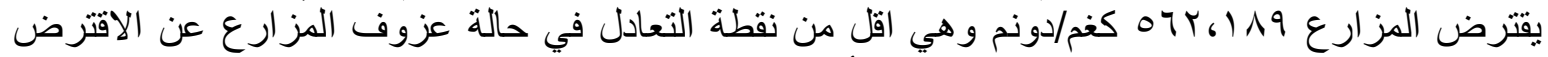

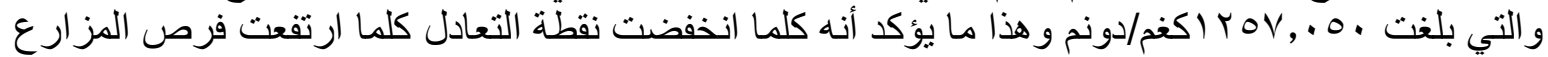

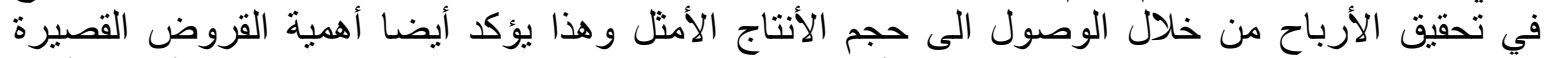

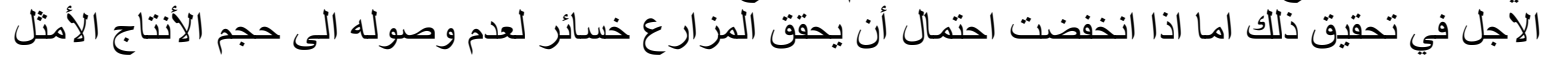

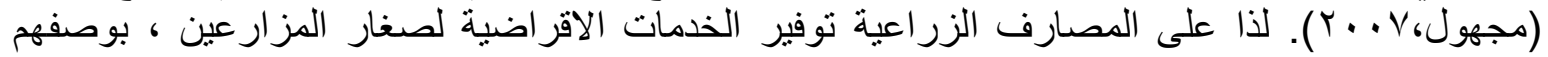

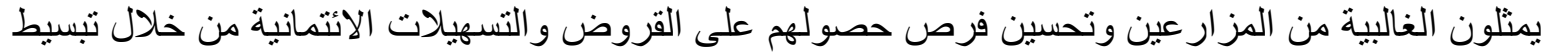

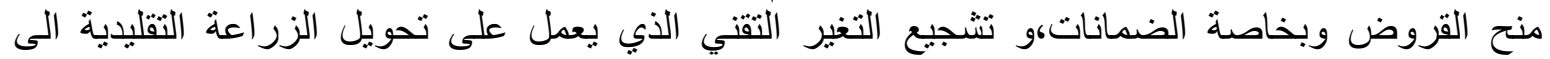

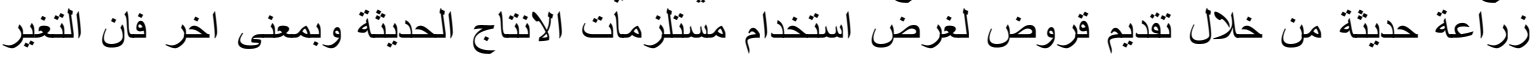

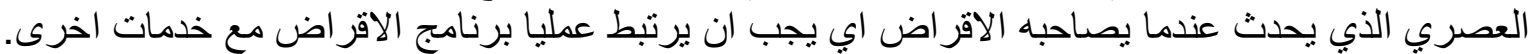

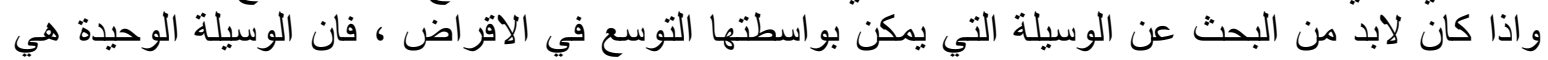

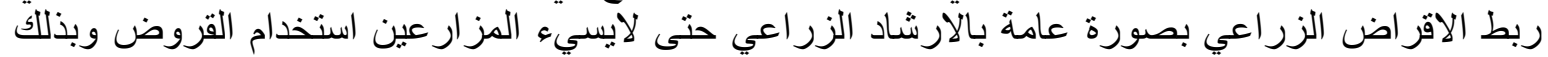

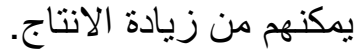

\title{
THE SHORT-TERM AGRICULTUAL LENDING OF ACCESS TO THE OPTIMAL PRODUCTION VOLUME FOR WHEAT FARMERS IN NINEVEH GOVERNORATE
}

\author{
Hanaa' S. dawood
}

Agriculture Economic Dept/ Agriculture \& Forestry College /Mousl University

\begin{abstract}
The research aims to quantify the impact of short-term loans in determining amazed optimal output achieved at the lowest point of the average total costs at the farm level. The search started from hypothesis that small producers of wheat liable variable capital necessary for expansion in investment the variable elements production to arrive rational stage of production. And comes the role of short-term borrowing and one of the tools to help producers to achieve this. They designed a questionnaire included a series of questions related to various production costs and what can leave over the farmer from the production requirements in case of the
\end{abstract}


lack of capital and also he does not borrowed The sample included research (50) farms from farmers wheat crop in the area of Qush. One of the most important findings of the research show that the productivity of one ton of the wheat crop in the case of farmer do not borrowing was $558.622 \mathrm{~kg}$ while the productivity of one ton in the case of farmer borrowing was $1091 \mathrm{~kg}$. It included some of the recommendations.

\section{المصادر}

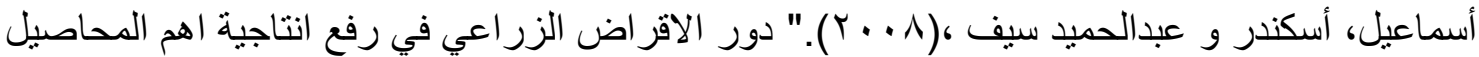

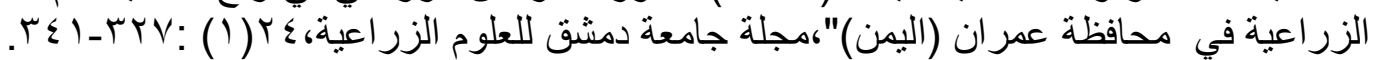

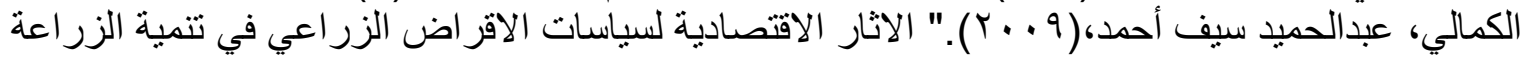

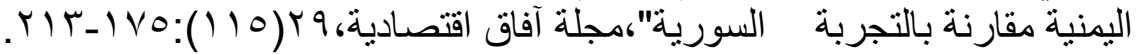

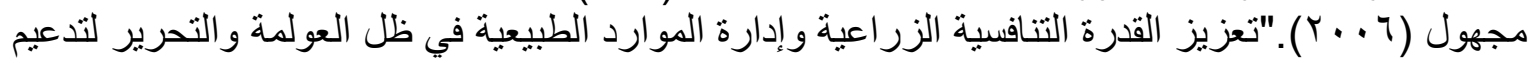

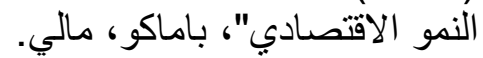

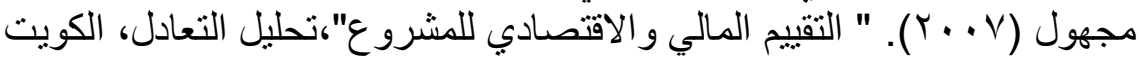

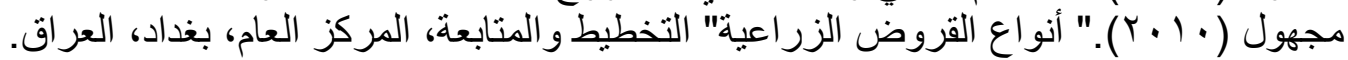

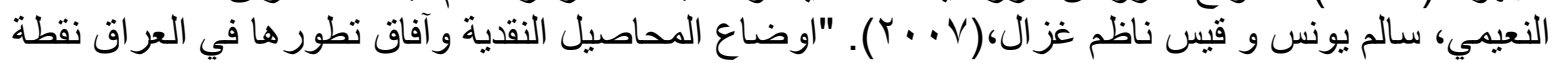

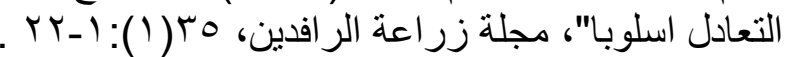

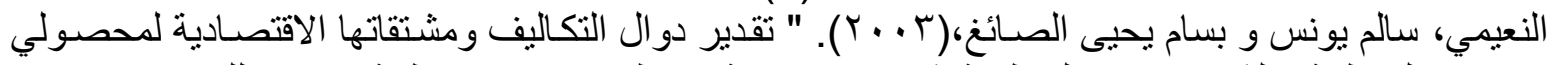

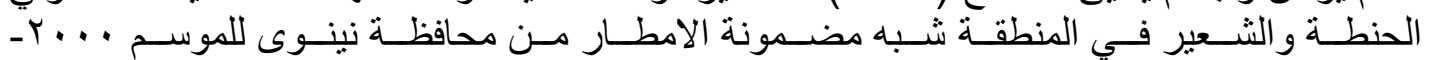

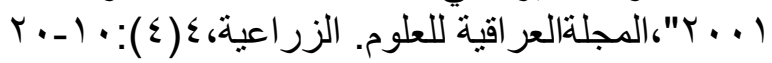

Ahmad,Nawaz,(2011).Impact of institutional credit on agricultural output a case study of Pakistan, Jour. Theoretical and Applied Economics, 10(563):99-120

Chowdhury, Tanbir Ahmad(2011).Performance evaluation of agricultural banks in Bangladesh, International journal business and management, 6(4):75-89.

Dong, Fengxia,(2010). Effects of credit constraint on productivity and rural household income in China, Jour. Agricultural and applied Economics association, 14:45-64

Owuor, George,(2009). Based credit uphold smallholder farmers productivity and reduce Poverty in Africa- empirical evidence from Kenya, Jour. Micro finance 8(20):111-128.

Simtowe, Franklin and Manfred Zeller,(2006). The impact of access to credit on the adoption of hybrid maize in Malawi, Jour. Agricultural economics research review association,23:165-171.

Wang, Yun-Kui,(2010). An analysis of evolution of lending patterns of IFAD China's rural financial project, Asian Agricultural research,2(3):5-14 